Classification

Physics Abstracts

$05.70 \mathrm{~F}-87.20$

\title{
Budding of membranes induced by intramembrane domains
}

\author{
Reinhard Lipowsky \\ Institut für Festkörperforschung, Forschungszentrum Jülich, Postfach 1913, D-5170 Jülich, \\ Germany
}

(Received 26 June 1992, accepted 17 July 1992)

\begin{abstract}
Membranes are often composed of a mixture of amphiphilic molecules which aggregate into clusters or domains. A simple theoretical model is introduced which predicts that flat or weakly curved domains become unstable at a certain limiting size and then undergo a budding or invagination process. This shape transformation is primarily driven by the line tension of the domain edge. It is also predicted that the budding domain can rupture the membrane and then pinch off from the matrix. The size of the bud and the time scales involved in the budding dynamics are estimated for model membranes composed of lipid mixtures. This instability mechanism should also be effective for the budding of biomembranes.
\end{abstract}

\section{Introduction.}

Membranes such as lipid bilayers are highly flexible and thus can easily change their shape. Recently, a variety of shape transformations have been observed by phase contrast microscopy of giant lipid vesicles [1-7]. It was found that these vesicles can exhibit budding or invagination processes in which small vesicles bud off from a larger membrane surface. These processes can be simply induced by a change in temperature. Such temperature-induced budding can be understood theoretically if one assumes that the lipid bilayer of the vesicle is laterally homogeneous. Its shape is then determined, to a large extent, by the area to volume ratio. The thermal expansivity of the bilayer membrane is large compared to that of the water. Therefore, as the temperature is increased, the bilayer membrane expands more rapidly than the enclosed water, and the area to volume ratio of the vesicles is increased.

In biological cells, budding is a rather frequent event since it represents the first step in the production of transport vesicles which shuttle between different compartments of the cell [8-11]. Two budding processes can be distinguished: (i) Endocytosis of the plasma membrane, i.e., budding of the plasma membrane towards the interior of the cell; and (ii) budding of the membranes bounding internal compartments such as the endoplasmic reticulum, the stack of Golgi cisternae, and the trans Golgi network. In the latter case, the budding vesicle points towards the exterior of the internal compartment. 


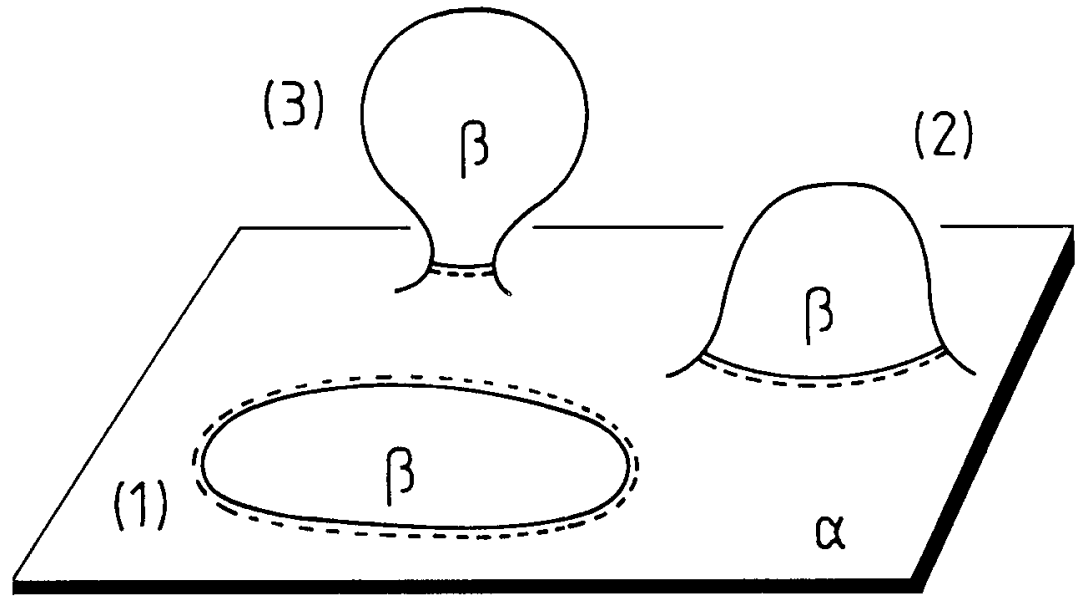

Fig.1. - Budding of the membrane domain $\beta$ embedded in the membrane matrix $\alpha$. The domain edge is indicated by the full-broken line. The length of this edge decreases during the budding process from (1) to (3).

Even though the budding of biomembranes resembles the temperature-induced shape transformations of lipid vesicles, the underlying mechanism must be quite different. Biomembranes are composed of many different lipids and proteins which diffuse laterally along the membrane and can therefore aggregate into clusters or domains. Indeed, the budding of biomembranes is believed to be preceded by the formation of such intramembrane domains [8-11].

It is shown in this paper that a domain within a fluid membrane should always undergo a budding process as soon as it has attained a certain size. This process is induced by the line tension of the domain edge: as can be seen by inspection of figure 1, the edge energy of the budded domain is much smaller than the edge energy of the flat (or weakly curved) domain. More precisely, the instability of the flat domain is governed by the competition between the line tension of the domain edge and the bending energy of the domain. This interplay will be studied in section 2 within a simplified theoretical model. In section 3, the dynamics of the budding process will be discussed. The various length and time scales involved in the process will be estimated for lipid bilayers in section 4. Finally, it is argued in section 5 that this mechanism should also be effective for biomembranes.

A variety of theoretical models for budding have been previously described in the literature. $[12,13]$ It seems, however, that the important role played by the line tension of the domain edge has been overlooked so far.

\section{Energy of budding domain.}

2.1 Edge ENERgy Versus BENDing ENERGy. - A flat domain with surface area $A$ embedded in a flat membrane matrix will form a circular disk of radius $L=(A / \pi)^{1 / 2}$ in order to attain a state with the minimal value, $2 \pi L$, for the length of its edge. However, as far as the edge energy is concerned, a flat circular disk does not represent the state of lowest energy since the length of the edge can be further reduced if the domain deforms into the "third dimension" and forms a bud, see figure. 1.

Such a deformation necessarily involves an increase in the curvature and thus in the bending energy of the domain. The domain has minimal bending energy if its curvature is equal to the 


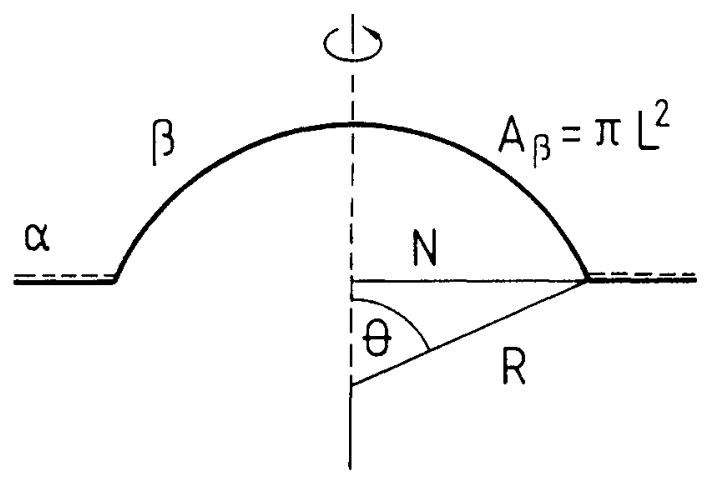

Fig.2. - Bud of $\beta$ domain forming a spherical cap. The area of the bud is $A_{\beta}=\pi L^{2}$. The bud has curvature $C=1 / R$ and neck radius $N$

spontaneous curvature, $C_{\mathrm{sp}}$. As mentioned, I will focus on the case offluid domains which do not build up a shear stress. In this case, the only elastic contributions to the energy of the budding domain are given by the edge and the bending energy.

2.2 A SIMPLIFIED MODEL. - Now assume that the domain forms a spherical cap with curvature $C$ and curvature radius, $R=1 / C$ as shown in figure 2 . The curvature $C$ can be positive or negative which allows to distinguish the two sides of the membrane. Here and below, an "up" bud has positive and a "down" bud has negative curvature. The spherical cap has a neck, the radius of which is denoted by $N$, see figure 2 .

The bending energy of the domain with surface area $A=\pi L^{2}$ is given by

$$
E_{\mathrm{bend}}=A \frac{1}{2} \kappa\left(2 C-2 C_{\mathrm{sp}}\right)^{2}=2 \pi \kappa\left(L C-L C_{\mathrm{sp}}\right)^{2}
$$

where $\kappa$ is the bending rigidity which has units of energy. The spontaneous curvature $C_{\mathrm{sp}}$ is zero if both sides of the membrane are identical. The expression (2.1) has to be supplemented by a small scale cutoff which is of the order of the membrane thickness, $a_{\text {mem }}$. Thus, the largest values for the curvatures $C$ and $C_{\mathrm{sp}}$ which can be treated in the framework of this model are of the order of $1 / a_{\text {mem }} \simeq 0.25 \mathrm{~nm}^{-1}$

The edge energy, on the other hand, is determined by the length, $2 \pi N$, of the edge. For the simple geometry considered here, one has $\sin \theta=2 \sin (\theta / 2) \cos (\theta / 2)=N / R=N C$ where the angle $\theta$ is defined in figure 2. The area of the spherical cap is given by $A=\pi L^{2}=$ $2 \pi R^{2}(1-\cos \theta)=4 \pi R^{2} \sin ^{2}(\theta / 2)$ which implies $\sin (\theta / 2)=L / 2 R=L C / 2$. A combination of these two relations leads to $N=L \sqrt{1-(L C / 2)^{2}}$. Therefore, the edge energy is given by

$$
E_{\text {edge }}=\sigma 2 \pi N=\sigma 2 \pi L \sqrt{1-(L C / 2)^{2}}
$$

where $\sigma$ denotes the line tension. If the bud forms a complete sphere, one has $L C= \pm 2$ and thus $E_{\text {edge }}=0$

It is convenient to introduce the characteristic invagination length, $\xi \equiv \kappa / \sigma$, which describes the interplay between the bending rigidity and the line tension, and the dimensionless energy $\vec{E} \equiv\left(E_{\text {bend }}+E_{\text {edge }}\right) / 2 \pi \kappa$. Inserting the expressions (2.1) and (2.2) for $E_{\text {bend }}$ and $E_{\text {edge, one }}$ obtains

$$
\bar{E}=\left(L C-L C_{\mathrm{sp}}\right)^{2}+(L / \xi) \sqrt{1-(L C / 2)^{2}}
$$




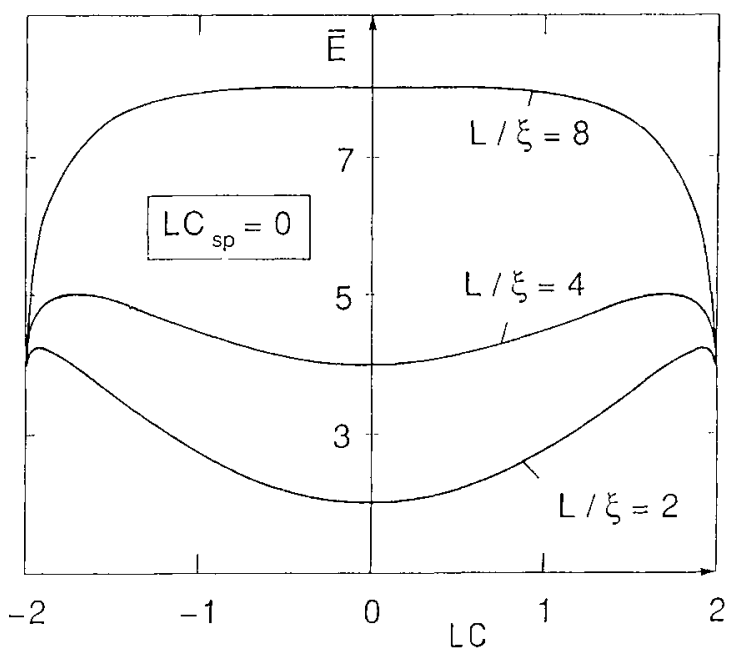

a)

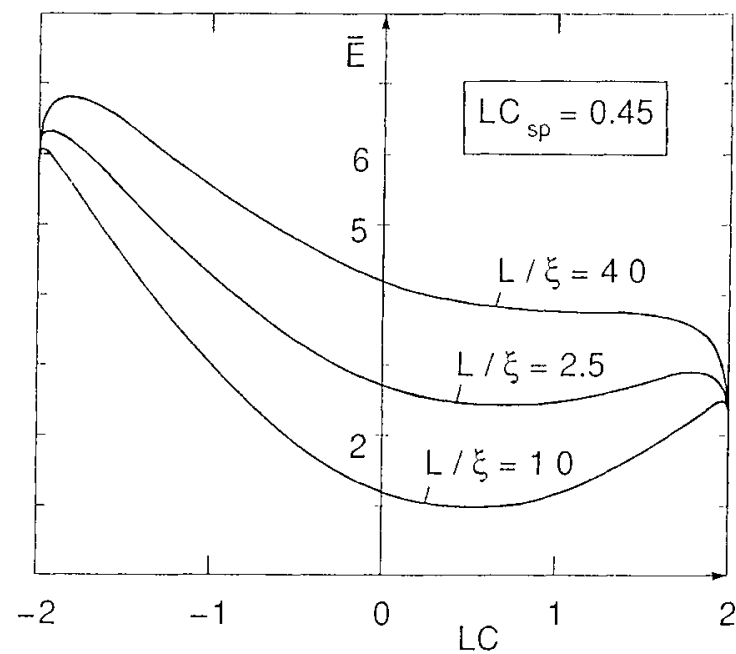

b)

Fig.3. - Reduced energy $\bar{E}$ of the budding domain as a function of the reduced curvature $L C$ : (a) for zero spontaneous curvature, $C_{\mathrm{sp}}=\mathbf{0}$, and (b) for nonzero spontaneous curvature with $L C_{\mathrm{sp}}=\mathbf{0 . 4 5}$ The length scale $L$ denotes the linear size of the domain; the invagination length $\xi=\kappa / \sigma$, where $\kappa$ and $\sigma$ are the bending rigidity and the line tension of the domain edge. The uppermost curve corresponds to the size $L=L^{\circ}$ at which the incomplete bud becomes unstable.

For zero spontaneous curvature, $C_{\mathrm{sp}}=0$, such a model has been previously used to discuss the size of vesicles generated by sonification [14].

In figure 3 , the functional dependence of the reduced energy $\vec{E}$ on the reduced curvature $L C$ is shown for zero and finite spontaneous curvature and for three values of $L / \xi$. It has been tacitly assumed here that there is no external tension acting on the membrane matrix. It will be shown below that the domain exerts a tension on the matrix during the budding process but that this tension relaxes relatively fast.

2.3 COMPLete AND INCOMPLETE BUDS. - As can be seen by inspection of figure 3, the energy $\bar{E}$ has several minima and maxima as a function of $L C$. There are always two boundary minima at $L C= \pm 2$ corresponding to the complete "up" and to the complete "down" sphere, respectively. The complete sphere with the lower energy will be called the complete bud. For zero spontaneous curvature, $C_{\mathrm{sp}}=0$, both complete spheres have the same energy, see figure $3 \mathrm{a}$, and the complete bud can develop equally well on both sides of the membrane: there is no difference between an "up" and a "down" bud. A finite value of $C_{\mathrm{sp}}$ breaks this symmetry, see figure $3 \mathrm{~b}$, and budding occurs preferentially on one side of the membrane.

The curvature radius of the complete bud will be denoted by $R_{\mathrm{cb}}=1 / C_{\mathrm{cb}}$; its absolute value is $\left|R_{\mathrm{cb}}\right|=L / 2$. Within the elastic model considered here, the complete bud is a limiting shape with zero neck radius, $N_{\mathrm{cb}}=0$. In practise, this neck will have a radius of the order of the membrane thickness, $N_{\mathrm{cb}} \simeq a_{\mathrm{mem}}$, as long as it does not break off from the matrix.

For small values of $L / \xi$, the energy $\bar{E}$ also exhibits a minimum at intermediate values of $L C$, see figure 3. The state corresponding to this minimum will be called the incomplete bud with curvature radius $R_{\mathrm{ib}}$ and finite neck radius $N_{\mathrm{ib}}$. For $C_{\mathrm{sp}}=0$, this minimum is at $L C=0$, see figure $3 \mathrm{a}$, and the so-called incomplete bud is not curved at all but flat with zero curvature and maximal neck radius $N_{\mathrm{1b}}=L$ 
In the following, the domain will be characterized by fixed elastic parameters $\kappa, C_{\mathrm{sp}}$ and $\sigma$, and thus by the fixed invagination length $\xi=\kappa / \sigma$. The domain size $L$, on the other hand, changes because the domain grows in time by some aggregation process within the membrane. Therefore, the domain size $L$ will play the role of a control parameter for the budding process.

For $L \ll \xi$, the energy $\bar{E}$ has the functional forms as given by the bottom curves in figures 3a and $3 \mathrm{~b}$. In this case, the domain forms an incomplete bud corresponding to the minimum of $\bar{E}$ at intermediate $L C$-values. This state has the lowest energy and thus represents the stable state of the domain since the cost in bending energy is larger than the possible gain in edge energy. As $L$ grows, the edge of the domain becomes longer, and the energy of the incomplete bud is increased.

2.4 CoeXistence of INCOMPLete AND COMPLETE BUD. - At a certain critical size, $L=L^{*}$, the energy, $\bar{E}_{1 \mathrm{~b}}$ of the incomplete bud becomes equal to the energy $\bar{E}_{\mathrm{cb}}$ of the complete bud. This situation corresponds to the middle curves of $\bar{E}$ in figures $3 \mathrm{a}$ and $3 \mathrm{~b}$.

It follows from the expression (2.3) for the dimensionless energy $\bar{E}$ that the critical size $L^{*}$ behaves as

$$
L^{*} \approx \frac{4 \xi}{1+4 \xi\left|C_{\mathrm{sp}}\right|} \quad \text { for small } \xi\left|C_{\mathrm{sp}}\right|
$$

and as

$$
L^{*} \approx \frac{2}{\left|C_{\mathrm{sp}}\right|}\left\{1-\frac{3}{4}\left(\xi\left|C_{\mathrm{sp}}\right|\right)^{-2 / 3}\right\} \quad \text { for large } \xi\left|C_{\mathrm{sp}}\right| .
$$

For $L=L^{*}$, the curvature, $C_{1 \mathrm{~b}}^{*}$, of the incomplete bud is given by

$$
\left|C_{\mathrm{ib}}^{*}\right| \approx \frac{2\left|C_{\mathrm{sp}}\right|}{1+4 \xi\left|C_{\mathrm{sp}}\right|} \quad \text { for small } \xi\left|C_{\mathrm{sp}}\right|
$$

and by

$$
\left|C_{\mathrm{ib}}^{*}\right| \approx\left|C_{\mathrm{sp}}\right|\left\{1+\frac{1}{4}\left(\xi\left|C_{\mathrm{sp}}\right|\right)^{-2 / 3}\right\} \quad \text { for large } \xi\left|C_{\mathrm{sp}}\right| .
$$

Likewise, the neck radius, $N_{\mathrm{lb}}^{*}$, of the incomplete bud has the asymptotic behavior

$$
N_{\mathrm{lb}}^{*} \approx L^{*}\left\{1-\frac{1}{2}\left(4 \xi\left|C_{\mathrm{sp}}\right|\right)^{2}\right\} \quad \text { for small } \xi\left|C_{\mathrm{sp}}\right|
$$

and

$$
N_{1 \mathrm{~b}}^{*} \approx 2 / \xi^{1 / 3}\left|C_{\mathrm{sp}}\right|^{4 / 3} \quad \text { for large } \xi\left|C_{\mathrm{sp}}\right| .
$$

Inspection of figure 3 shows that the two minima for the incomplete and for the complete bud are separated by an energy barrier, $\Delta \bar{E}^{*}=\bar{E}_{\max }^{*}-\bar{E}_{\mathrm{lb}}^{*}$, where $\bar{E}_{\max }$ is the energy of the intermediate maximum. This energy barrier behaves as

$$
\Delta \bar{E}^{*} \approx 1-2(\sqrt{3}-1) L^{*}\left|C_{\mathrm{sp}}\right| \quad \text { for small } \xi\left|C_{\mathrm{sp}}\right|
$$

and as

$$
\Delta \bar{E}^{*} \approx\left(\gamma^{2}-3 \gamma+2 \sqrt{\gamma}\right)\left(\xi\left|C_{\mathrm{sp}}\right|\right)^{-4 / 3} \quad \text { for large } \xi\left|C_{\mathrm{sp}}\right|
$$

with $\gamma=(2-\sqrt{3}) / 2$ and $\gamma^{2}-3 \gamma+2 \sqrt{\gamma} \simeq 0.35$. Both, the critical domain size $L^{*}$ and the associated energy barrier $\Delta \bar{E}^{*}$ take on their largest values for zero spontaneous curvature, compare figure 3 .

For $L=L^{*}$, the incomplete bud can be thermally activated to transform into the complete bud. The time scale for these activated processes is proportional to $\exp \left[\Delta E^{*} / T\right]=$ 
$\exp \left[2 \pi \kappa \Delta \bar{E}^{*} / T\right]$ and these processes are rare fluctuations as long as $\Delta \bar{E}^{*} \gg T / 2 \pi \kappa$. In the following, I will focus on the case of relatively large $\kappa / T$ as appropriate for lipid bilayers. Indeed, these bilayers are characterized by $T / 2 \pi \kappa \simeq 10^{-2}$. Using the asymptotic behavior as given by (2.11), one then finds that activated processes are rare fluctuations as long as $\xi\left|C_{\mathrm{sp}}\right| \ll 70$. This inequality is fulfilled for all examples considered below, see table I. In such a situation, the domain will stay in the incomplete bud state even as $L$ exceeds $L^{*}$

Table I. - Various length and time scales as appropriate for lipid bilayers characterized by spontaneous curvature $C_{\mathrm{sp}}$ and invagination length $\xi$.

\begin{tabular}{|cc|ccc|ccc|}
\hline$\left|C_{\mathrm{sp}}\right|$ & {$\left[\mathrm{nm}^{-1}\right]$} & 0 & $1 / 80$ & $1 / 30$ & 0 & $1 / 80$ & $1 / 30$ \\
$\xi$ & {$[\mathrm{nm}]$} & 10 & 20 & 20 & 100 & 200 & 200 \\
\hline $4 \xi\left|C_{\mathrm{sp}}\right|$ & & 0 & 1 & 2.7 & 0 & 10 & 27 \\
\hline$L^{\circ}$ & {$[\mathrm{nm}]$} & 80 & 57 & 32 & 800 & 160 & 51 \\
$N_{\mathrm{bb}}^{\circ}$ & {$[\mathrm{nm}]$} & 80 & 40 & 19 & 800 & 50 & 16 \\
$R_{\mathrm{cb}}^{\circ}$ & {$[\mathrm{nm}]$} & 40 & 28 & 16 & 400 & 60 & 25 \\
\hline$t_{\mathrm{d}}$ & {$[\mathrm{s}]$} & $10^{-2}$ & $10^{-3}$ & $10^{-3}$ & 1 & $10^{-2}$ & $10^{-3}$ \\
$t_{\mathrm{s}}$ & {$[\mathrm{s}]$} & $10^{-8}$ & $10^{-9}$ & $10^{-10}$ & $10^{-6}$ & $10^{-9}$ & $10^{-10}$ \\
$t_{\mathrm{f}}$ & {$[\mathrm{s}]$} & $10^{-1}$ & $10^{-2}$ & $10^{-3}$ & $10^{2}$ & $10^{-2}$ & $10^{-3}$ \\
\hline
\end{tabular}

2.5 Instability of InCOMPlete BUD. - For domain size $L>L^{*}$, the incomplete bud represents a metastable or locally stable state up to the limiting size $L=L^{\circ}$ at which the energy barrier disappears and the incomplete bud becomes unstable [15]. This situation corresponds to the top $\bar{E}$-curves in figures 3 a and 3 b.

It follows from the expression (2.3) for the energy $\bar{E}$ that the limiting size $L^{\circ}$ is given by

$$
L^{\circ}=8 \xi /\left[1+\left(4 \xi\left|C_{\mathrm{sp}}\right|\right)^{2 / 3}\right]^{3 / 2}
$$

which is valid for all values of $\xi\left|C_{\mathrm{sp}}\right|$. As the incomplete bud becomes unstable, it is characterized by the curvature

$$
C_{\mathrm{ib}}^{\circ}=C_{\mathrm{sp}}\left\{1+\left(4 \xi\left|C_{\mathrm{sp}}\right|\right)^{-2 / 3}\right\}
$$

and by the neck radius

$$
N_{\mathrm{ib}}^{\mathrm{o}}=8 \xi /\left[1+\left(4 \xi\left|C_{\mathrm{sp}}\right|\right)^{2 / 3}\right]^{2} .
$$

The difference, $\delta \bar{E}^{\circ}=\bar{E}_{\mathrm{bb}}^{\circ}-\bar{E}_{\mathrm{cb}}^{\circ}$, between the energies of the incomplete and the complete bud represents the amount of work which the budding domain can do on the rest of the system. This energy difference is given by

$$
\delta \bar{E}^{\circ}=-4(q / 8)^{2 / 3}+q(q / 8)^{1 / 3}+q\left[(8 / q)^{2 / 3}-1\right]^{3 / 2}\left[1-\sqrt{1-(q / 8)^{2 / 3}}\right]
$$


with $q \equiv L^{\circ} / \xi$ and $L^{\circ}$ as given by (2.12). For zero spontaneous curvature, the incomplete bud is flat and $N_{\mathrm{ib}}^{\mathrm{o}}=L^{\circ}$. In this case, one has $q=L^{\circ} / \xi=8$ and the reduced energy difference $\delta \bar{E}^{\circ}$ is given by $\delta \bar{E}^{\circ}=4$. For $4 \xi\left|C_{\mathrm{sp}}\right|=1$, one has $R_{\mathrm{ib}}^{\mathrm{o}}=N_{\mathrm{bb}}^{\mathrm{o}}$, and the incomplete bud forms a hemisphere which is now transformed into a complete sphere. In the latter case, one has $\delta \bar{E}^{\circ}=2(\sqrt{2}-1)=0.83$

In the next section, the time scales for the dynamics of budding will be estimated. One then finds that the domain size remains essentially constant during the transformation from the incomplete to the complete bud. This implies that the complete bud has curvature radius $\left|R_{\mathrm{cb}}^{\mathrm{o}}\right|=L^{\circ} / 2$. It then follows from (2.12) that its curvature $C_{\mathrm{cb}}=1 / R_{\mathrm{cb}}$ is given by

$$
\begin{aligned}
C_{\mathrm{cb}}^{\circ} & =C_{\mathrm{sp}}\left\{1+\left(4 \xi\left|C_{\mathrm{sp}}\right|\right)^{-2 / 3}\right\}^{3 / 2} \\
& =C_{\mathrm{ib}}^{o}\left\{1+\left(4 \xi\left|C_{\mathrm{sp}}\right|\right)^{-2 / 3}\right\}^{1 / 2}
\end{aligned}
$$

\section{Dynamics of budding.}

The simple model discussed above reveals that the budding process involves several steps. First, the domain forms an incomplete bud which grows by diffusion-limited aggregation up to a certain limiting size. At this point, the incomplete bud becomes unstable and is then transformed into a complete bud [15]. The relative contribution of these two steps to the curvature of the domain is determined by the dimensionless ratio $4 \xi\left|C_{\mathrm{sp}}\right|=4 \kappa\left|C_{\mathrm{sp}}\right| / \sigma$. For relatively small spontaneous curvature $C_{\mathrm{sp}}$ or relatively large line tension $\sigma$, the incomplete bud is flat or weakly curved and the bud has to acquire most of its curvature during the transformation step. For relatively large spontaneous curvature or relatively small edge tension, on the other hand, the curvature of the bud builds up already during the growth step. In addition, there can be another step in which the neck breaks off from the matrix and the budding domain becomes a separate vesicle.

3.1 GROWTH OF INCOMPLETE BUD. - First, let us consider the growth step. As mentioned, the intramembrane domain grows via the aggregation of molecules which diffuse laterally within the membrane matrix. If one can ignore interactions between different domains, the size $L$ of a single domain grows as $L \sim(D t)^{1 / 2}$ with time $t$ where $D$ is the diffusion coefficient of the molecules. It then takes a time of the order of

$$
t_{\mathrm{d}} \sim L^{2} / D
$$

until the domain has grown up to size $L$. In this estimate, the nucleation time for the domain formation is not included.

If the membrane contains many domains, the interaction of these domains can lead to various growth mechanisms. If the collective diffusion of the domain within the membrane is relatively fast, the domains will collide and can then grow by coalescence. On the other hand, if the domain diffusion is relatively slow, the growth could be governed by the evaporationcondensation mechanism of Lifshitz-Slyozov in which the growth rate is limited by the diffusive transport from small to large domains. If the growth is dominated by coalescence, the domain size $L$ will again grow as $L \sim t^{1 / 2}$ with time $t$ [16]. In contrast, the Lifshitz-Slyozov mechanism leads to $L \sim t^{1 / 3}[17]$.

3.2 TRANSFORMATION FROM INCOMPLETE TO COMPLETE BUD. - Next, consider the transformation step from the incomplete to the complete bud. During this step, the domain has to pull in membrane area. For the bud geometry considered here, this area, $\delta A$, is equal to 
$\delta A=\pi N^{2}$ where $N$ is the neck radius of the incomplete bud. In principle, a variety of area reservoirs could be accessible to the budding domain such as e.g. adhering vesicles which fuse with the membrane or membrane foldings and pockets. For simplicity, let us focus on the case where the membrane matrix surrounding the bud is essentially flat but exhibits thermallyexcited undulations. In this situation, the transforming bud can pull in the excess area stored in the undulations or pull in area by stretching the matrix surrounding it.

In both cases, the bud perturbs a certain minimal size $L_{1}$ of the matrix. As shown in appendix A, the matrix segment stretched by the bud has a linear size $L_{1}$ which can be estimated as

$$
L_{1} \simeq\left(K_{\mathrm{A}} / 4 \kappa \delta \bar{E}^{\circ}\right)^{1 / 2} N^{2}
$$

with $N=N_{\mathrm{ib}}^{\circ}$ as given by (2.14). The parameter $K_{\mathrm{A}}$ denotes the area compressibility modulus. This stretching process takes a time $t_{\mathrm{s}}$ which is of the order of

$$
t_{\mathrm{s}} \sim\left(\rho_{\mathrm{A}} / K_{\mathrm{A}}\right)^{1 / 2} L_{1}
$$

where $\rho_{\mathrm{A}}$ is the mass density of the membrane per unit area.

On the other hand, the bud may also pull in area if it flattens the undulations of the surrounding matrix. As shown in appendix A, it then perturbs a matrix segment of linear size

$$
L_{1} \approx\left[8 \pi \kappa / T \ln \left(1+2 \delta E^{\circ} / \pi T\right)\right]^{1 / 2} N
$$

with neck radius $N=N_{\mathrm{bb}}^{\mathrm{o}}$ of the incomplete bud. This flattening process takes a time $t_{\mathrm{f}}$ which is of the order of

$$
t_{\mathrm{f}} \sim \eta L_{1}^{3} / \kappa
$$

where $\eta$ denotes the dynamic viscosity of the aqueous solution.

3.3 Detachment of Budding Domain. - Up to now, it has been tacitly assumed that the bud remains connected to the membrane matrix by a narrow neck. However, during the transformation towards the complete bud, the neck radius decreases which implies that the lateral tension exerted by the bud onto the surrounding matrix along the domain edge increases. Within the model considered here, the tension $\Sigma$ along the edge of the domain, i.e., along the neck of the bud is given by

$$
\Sigma=(1 / 2 \pi N)(\partial E / \partial N)=(\sigma / N)-8 \kappa\left(1-C_{\mathrm{sp}} / C\right) / L^{2}
$$

where the curvature $C$ depends on the neck radius $N$ via $(L C / 2)^{2}=1-(N / L)^{2}$

By definition, the lateral tension $\Sigma$ is positive if the surrounding matrix is pulled by the bud, and negative if the bud is pulled by the matrix. In figure 2, those segments of the $\bar{E}$-curves which have negative slope correspond to a positive lateral tension.

During the transformation step, the neck narrows down and the tension $\Sigma$ as given by (3.6) becomes larger. As the neck attains a certain radius, this tension may exceed the tension of rupture, $\Sigma_{\max }$, of the domain edge. Since the edge represents a linear defect (with a finite line tension), its tension of rupture is smaller than the tension of rupture for the rest of the membrane. For $\Sigma \simeq \Sigma_{\max }$, the bud pulls so strongly that the neck breaks off and the budding domain becomes a budding vesicle. In this way, the action of the line tension provides a general mechanism for the detachment of the budding domain.

If the membrane contains many budding domains, each bud will experience an effective lateral tension, $\Sigma_{\text {eff }}<0$, arising from the completion of other buds. In the presence of an 
external lateral tension $\Sigma$, the energy of the bud is given by $E_{\Sigma}=E+\pi N^{2} \Sigma$ where $\pi N^{2}$ is the area of the neck of the bud. Using the relation $N^{2}=L^{2}\left(1-(L C / 2)^{2}\right)$, one obtains the dimensionless energy

$$
\bar{E}_{\Sigma}=\bar{E}+\left(L^{2} \Sigma / 2 \kappa\right)-\left(L^{2} \Sigma / 8 \kappa\right)(L C)^{2}
$$

For $\Sigma=\Sigma_{\text {eff }}<0$, this tension acts to stabilize the incomplete bud and thus to prolong the budding process. For example, a domain with zero spontaneous curvature now becomes unstable for the limiting size

$$
L^{\circ}=(\sigma / 2 \Sigma)\left(-1+\sqrt{1+32 \Sigma \xi^{2} / \kappa}\right)
$$

which behaves as

$$
L^{\circ} \approx 8 \xi\left(1-8 \Sigma \xi^{2} / \kappa\right) \text { for small } \Sigma \xi^{2} .
$$

In practice, the effective tension within the matrix arising from the buds will strongly fluctuate since it depends on the density and on the states of these buds.

\section{Budding of lipid bilayers.}

Budding induced by intramembrane domains could be studied experimentally in simple model membranes such as lipid bilayers which are composed of a mixture of lipids and exhibit a coexistence region between two phases. In most systems studied so far, one of the two phases was a gel or a polymerized state [18-21]. However, systems with two coexisting phases which are both fluid have also been identified. One prominent example is a mixture of phospholipids and cholesterol which exhibits a broad coexistence region for a fluid "ordered" and a fluid "disordered" phase [22-24]. Other examples are the binary mixture of DEPC and DPPE [25], and mixtures of phospholipids with partially unsaturated acyl chains [26].

First, consider a domain in the bilayer which extends across both monolayers. In this case, both monolayers of the domain have the same chemical composition and the bilayer has zero spontaneous curvature, $C_{\mathrm{sp}}=0$. Its bending rigidity $\kappa$ can be deduced from experimental observations on the shape fluctuations (or flickering) of vesicles; a typical value is $\kappa \simeq 10^{-19} \mathrm{~J}$ $[27,28]$. Finally, the line tension $\boldsymbol{\sigma}$ can be estimated as follows.

The edge of the bilayer domain represents a cut across the whole bilayer. The cross-section of such a cut consists of three distinct regions: two hydrophilic head-group regions of thickness $a_{\mathrm{h}} \simeq 1 \mathrm{~nm}$ and an intermediate hydrophobic tail region of thickness $a_{\mathrm{t}} \simeq 4 \mathrm{~nm}$. The headgroup region and the tail region can have distinct interfacial free energies per unit area, say $\Sigma_{\mathrm{h}}$ and $\Sigma_{\mathrm{t}}$. Then, the edge tension $\sigma$ can be estimated by $\sigma \simeq a_{\mathrm{h}} \Sigma_{\mathrm{n}}+a_{\mathrm{t}} \Sigma_{\mathrm{t}}$. For 3-dimensional fluid phases, a typical value for the interfacial free energy is $\Sigma \simeq 10^{-2} \mathrm{Jm}^{-2}$. If one assumes (i) that $\Sigma_{\mathrm{h}} \simeq \Sigma$ and (ii) that $\Sigma_{\mathrm{t}} \ll \Sigma_{\mathrm{h}}$ (as appropriate for two phases which have a similar hydrocarbon region), one obtains the crude estimate $\sigma \simeq a_{\mathrm{h}} \Sigma \simeq 10^{-17} \mathrm{~J} \mu \mathrm{m}^{-1}$

For the typical values $\kappa \simeq 10^{-19} \mathrm{~J}$ and $\sigma \simeq 10^{-17} \mathrm{~J} \mu \mathrm{m}^{-1}$, the invagination length $\xi=\kappa / \sigma \simeq$ $10 \mathrm{~nm}$. According to (2.6), the incomplete bud becomes unstable when it has the limiting size $L^{\circ}=8 \xi \simeq 80 \mathrm{~nm}$

For lipid molecules in fluid bilayers, a typical value for the diffusion coefficient $D$ is $D \simeq 10^{-12}$ $\mathrm{m}^{2} \mathrm{~s}^{-1}$ [29]. It then takes of the order of $t_{\mathrm{d}} \simeq L^{2} / D \simeq 10^{-2} \mathrm{~s}$ to grow a domain of size $L=L^{\circ}=80 \mathrm{~nm}$. When the domain has attained this size, it becomes unstable and transforms into a complete bud. The time scales for this transformation can be estimated from (3.2) (3.5). For lipid bilayers, the area compressibility modulus $K_{\mathrm{A}}$ has been measured to be of the order of $K_{\mathrm{A}} \simeq 10^{-19} \mathrm{~J} \mathrm{~nm}^{-2}$ [30], and the mass density $\rho_{\mathrm{A}}$ per unit area can be estimated to be of the order of $\rho_{\mathrm{A}} \simeq 3 \times 10^{-6} \mathrm{~kg} \mathrm{~m}^{-2}$. It then follows from (3.2) and (3.3) that it takes a time $t_{\mathrm{s}}$ of the order of $t_{\mathrm{s}} \simeq 10^{-8} \mathrm{~s}$ in order to pull out the area by stretching the matrix. 
The time scale $t_{\mathrm{f}}$ for the flattening of the undulations, on the other hand, depends on the dynamic viscosity $\eta$ of the surrounding medium as in (3.5). The dynamic viscosity of water is $\eta \simeq 10^{-3} \mathrm{~Pa} \mathrm{~s}=10^{-3} \mathrm{~kg} \mathrm{~m}^{-1} \mathrm{~s}^{-1}$. At room temperature, one has $\kappa / T \simeq 20$. It then follows from (3.4) and (3.5) that it takes a time $t_{\mathrm{f}}$ of the order of $t_{\mathrm{f}} \simeq 10^{-1} \mathrm{~s}$ in order to flatten the undulations. Thus, the transformation from the incomplete to the complete bud will first proceed by stretching the surrounding matrix. This induces a lateral tension within the membrane matrix which will then flatten the membrane undulations.

Since the time scale $t_{\mathrm{s}} \simeq 10^{-8} \mathrm{~s}$ is also very small compared to the diffusion time $t_{\mathrm{d}}$, the domain size stays essentially constant during the transformation step. This implies that the complete bud has the radius $R_{\mathrm{cb}}^{\mathrm{o}} \simeq L^{\circ} / 2=40 \mathrm{~nm}$ for the above choice of elastic parameters. The various length and time scales for the budding of a bilayer domain with $C_{\mathrm{sp}}=0$ are summarized in table I.

The line tension $\sigma$ is substantially reduced in two cases: (i) the lipid mixture exhibits a critical point at which $\sigma$ vanishes (this critical point belongs to the universality class of the 2-dimensional Ising model); and (ii) the lipid bilayer contains edge-active molecules which preferentially adsorb at the domain boundary. (This represents the two-dimensional analogue to the reduction of the interfacial free energy by surface-active molecules in three dimensions). In both cases, the edge tension could be reduced and the bud radius could be increased by more than an order of magnitude. For $\sigma \lesssim 10^{-18} \mathrm{~J} \mu \mathrm{m}^{-1}$, one obtains larger buds with $R_{\mathrm{cb}}^{\circ} \gtrsim 0.4 \mu \mathrm{m}$ which could be directly observed by optical microscopy.

Next, consider a domain within the bilayer which extends only across one monolayer. The line tension of such a monolayer domain is about half the line tension of a bilayer domain. The bending rigidity $\kappa$ will have the same order of magnitude, $\kappa \simeq 10^{-19} \mathrm{~J}$, as for the bilayer domain. These values for the elastic parameters lead to the invagination length $\xi \simeq 20 \mathrm{~nm}$

For a monolayer domain, the lipid composition in both monolayers is different, and the bilayer membrane can have a finite spontaneous curvature $C_{\mathrm{sp}} \neq 0$. This spontaneous curvature depends on the shape of the molecules and thus cannot be estimated in general. A rough idea about its magnitude can be obtained by comparison with surfactant mixtures in water which spontaneously form a dispersion of vesicles in full chemical equilibrium. For example, mixtures of two single-chained surfactants with oppositely charged head groups spontaneously form vesicles with a spontaneous curvature radius $R_{\mathrm{sp}}=1 / C_{\mathrm{sp}}$ which varies from 30 to $80 \mathrm{~nm}$ depending on the concentration of the surfactants [31]. If one assumes that these values also apply to a monolayer domain in a lipid bilayer (for which the exchange of molecules with the surrounding aqueous medium is extremely slow), one finds from (2.12) and $\xi \simeq 20 \mathrm{~nm}$ that the bud radius $R_{\mathrm{cb}}^{\circ}=L^{\circ} / 2$ varies from 16 to $28 \mathrm{~nm}$. These length scales and the corresponding time scales are also displayed in table I.

What is the lateral tension, $\Sigma=\Sigma_{\mathrm{cb}}$, across the neck of the complete bud? It turns out that in all cases discussed here this tension is primarily determined by the first term in (3.6), i.e., that $\Sigma \simeq \sigma / N_{\mathrm{cb}}$ with $N_{\mathrm{cb}} \simeq a_{\mathrm{mem}} \simeq 4 \mathrm{~nm}$. Using the above estimates for the line tension $\sigma$, one finds that $\Sigma_{\mathrm{cb}}$ is of the order of $10^{-3} \mathrm{~J} / \mathrm{m}^{2}$ for the bilayer membranes. It is important to note that this lateral tension is comparable to the tension of rupture, $\Sigma_{\max }$, for lipid bilayers which typically lies in the range $(1-5) \times 10^{-3} \mathrm{~J} / \mathrm{m}^{2}$ [30]. Thus, for lipid bilayers, the lateral tension exerted by the bud can be sufficient to break the neck of the complete bud and thus to pinch off the budding vesicles.

\section{Budding of biomembranes.}

As mentioned, budding of biomembranes represents the first step in the production of vesicles for intracellular transport. Two pathways are currently distinguished: (i) budding domains 
which are coated by a network of clathrin molecules; and (ii) budding domains which are not coated by such a network. In the latter case, the domain may have a coat which does not contain clathrin or it may have no coat at all. It is believed that clathrin-coated domains or pits provide the major pathway for endocytosis of the plasma membrane but that nonclathrin-coated domains are involved in most exocytic processes of internal membranes [11].

In general, the main function for clathrin and other coating proteins seems to be that it facilitates the uptake of receptors and ligands: there is "life without clathrin" even though it is less efficient [11]. It seems plausible to assume that the evolution of biomembranes progressed from budding without a network of coating proteins towards budding with such a network. In the absence of coating proteins, the budding mechanism of biomembranes should be induced by the formation of the receptor domain and thus should be governed by the interplay of the bending energy and the line tension of this domain as discussed above. How is this budding mechanism affected by the presence of coating proteins?

One important class of budding processes which involve a coat of assembled proteins is receptor-mediated endocytosis of the plasma membrane via clathrin-coated pits or domains. It is now generally established that many receptors which diffuse within the plasma membrane aggregate into such domains [10, 11]. Many of these receptors participate irrespective of whether they have bound their specific ligands. In addition, different kinds of receptors cluster in the same coated pit.

The elementary building blocks of the coat are receptor molecules with clathrin trimers (or triskelions) attached to receptor tails via assembly polypeptides. The clathrin molecule has three kinked legs extending from a central vertex. In aqueous solution, these trimers spontaneously assemble into polyhedral cages; at $\mathrm{pH}$ 6.2, these polyhedra consist of 140 clathrin molecules (which form 12 pentagons and 60 hexagons) and have a curvature radius, $R_{\mathrm{cl}} \simeq$ $60 \mathrm{~nm}$. Similar cages have been identified in various tissues: three distinct polyhedra with curvature radius $R_{\mathrm{cl}} \simeq 38,45$, and $60 \mathrm{~nm}$ consisting of 60,82 , and 140 clathrin molecules have been found in brain, liver and fibroblast cells, respectively [32].

The binding energy of the clathrin molecules within the polymerized network can be estimated from the depolymerization process which is regulated in the cell by special uncoating proteins. It seems that these proteins need to hydrolyse three ATP molecules in order to detach one clathrin trimer from the polymerized coat [29]. Each hydrolysed ATP molecule releases a free energy $\Delta G_{\mathrm{ATP}} \simeq 8 \times 10^{-20} \mathrm{~J} \simeq 20 k_{\mathrm{B}} T$ at room temperature. Thus, within the polymerized network, the binding energy per clathrin molecule seems to be $\simeq 60 k_{\mathrm{B}} T$

Originally, it was thought that the clathrin molecules adsorb onto the protein-lipid bilayer and first form a planar hexagonal network. However, because of the large binding energy involved in the polymerization, it would cost a lot of energy to disassemble and reassemble this network during the subsequent budding process. Thus, it seems plausible to assume that the molecules polymerize only once during the budding process and then form a network with curvature $C \simeq C_{\mathrm{cl}}$

If the building blocks had an appropriate conical shape which leads to a spontaneous curvature $C_{\mathrm{sp}}=C_{\mathrm{cl}}$, the polymerization could proceed at the growing edge of the domain. In this case, the domain starts off from a nucleated cluster with curvature $C \simeq C_{\mathrm{cl}}$, and each building block is incorporated into the polymerized network as it is attached to the growing domain. Since different receptors are assemblied within the same coated pit, the conical shape and the resulting spontaneous curvature must arise from the geometry of the receptor tails with the attached clathrin molecules (and assembly polypeptides).

On the other hand, the aggregating molecules could also have a spontaneous curvature, $C_{\mathrm{sp}}$, which is small compared to the curvature, $C_{\mathrm{cl}}$, of the polymerized clathrin cage provided the domain stays initially fluid. In the latter case, the budding of the domain would first be 
governed by the interplay of bending energy and line tension, and the polymerisation would only set in during the late stages of the budding process when the curvature of the budding domain attains the value $C \simeq C_{\mathrm{cl}}$

One should note that the two possible modes of polymerization just described are qualitatively different. If the building blocks are polymerized at the edge of the growing domain, the curvature of the domain will stay constant during the whole budding process. In contrast, if the polymerization is postponed until the curvature of the bud is compatible with the curvature of the clathrin cage, the domain curvature increases continuously during the budding process. For the endocytosis of large hen oocytes, electron microscopy seems to support the latter possibility since it indicates such a continuous increase of the domain curvature [8].

As long as the clathrin molecules form a polymerized network, the curvature of the bud remains fixed. In this way, the polymerization acts to select a certain curvature and thus to quantize the size of the budding vesicle. This could be advantageous for the subsequent transport and sorting of budded vesicles. In addition, the polymerized network also acts to stabilize the bud against the effective lateral tension exerted by competing buds. Indeed, in the absence of the polymerized coat, an almost complete bud could be flattened out again by this tension.

\section{Summary and outlook.}

In summary, a simple theoretical model has been introduced for domain-induced budding of membranes. This model predicts that domains should always undergo budding as soon as they have grown up to a certain limiting size $L^{\circ}$ as given by (2.12). This shape transformation is primarily driven by the line tension of the domain edge.

For bilayer membranes composed of lipid mixtures, the model makes definite predictions about the size of the bud and about the time scales involved in the budding process, see table I. These theoretical predictions are accessible to experiments. For example, one could study vesicles or multilayer suspensions composed of phosholipids and cholesterol. Initially, the concentration and the temperature are chosen in such a way that the bilayer membranes are in one of the two fluid phases and thus are laterally homogeneous. Then, a temperature quench is performed into the two-phase region where the two fluid phases can coexist. This will lead to the nucleation of intramembrane domains which should then undergo the budding process described here.

As discussed in section 5, the same mechanism should apply to the budding of non-coated domains in biomembranes. In fact, this mechanism could even be effective in the presence of coating proteins such as clathrin. In the latter case, the budding domain would stay unpolymerized until the curvature of the bud has attained a value which is compatible with the curvature of the polyhedral clathrin cage.

The simple model studied here has some obvious limitations. For example, it does not give an accurate description for the shape of the membrane close to the edge of the bud which should join smoothly with the larger membrane surface. This can be improved in a more refined theory in which the shape of the membrane is determined by a minimization procedure. Such an approach can also be used to incorporate the effect of global volume/area constraints on domain-induced budding of vesicles. In addition, one may study the effects of the Gaussian curvature term. Work on such a refined theory is in progress. 


\section{Acknowledgements.}

I thank Erich Sackmann, Willi Fenzl and Frank Jülicher for stimulating discussions.

\section{Appendix A. Time scales for transformation step.}

Consider a segment of area $A_{1}=\pi L_{1}^{2}$ of the membrane matrix surrounding the domain. In order to pull out the area $\delta A_{1}$ from this segment, the bud has to perform a certain amount of work, $\delta F_{1}$. In this appendix, this work is first determined for the process in which the area is pulled out by stretching the surrounding membrane, and secondly for the case in which the area is pulled out of the surrounding membrane by flattening its undulations. In both cases, $\delta F_{1}$ decreases monotonically with increasing size $L_{1}$ of the segment.

The maximal work which the transforming bud with $L=L^{\circ}$ as in (2.12) can do is given by $\delta E^{\circ}$. Obviously, one must have $\delta F_{1} \lesssim \delta E^{\circ}$. This implies a certain minimal value for the size $L_{1}$ of the membrane segment which is necessarily perturbed by the transforming bud. From this minimal size $L_{1}$, one can estimate the time scale for the transformation step.

This minimal value for $L_{1}$ follows from the two relations

$$
\delta A_{1}=\pi N^{2} \quad \text { and } \quad \delta F_{1}=\delta E^{\circ}=2 \pi \kappa \delta \bar{E}^{\circ} .
$$

Throughout this section, $N$ denotes the neck radius $N_{\mathrm{bb}}^{\mathrm{o}}$ of the incomplete bud as given by (2.14).

A1. STRETChING OF SURROUNDING MATRIX.- The work necessary to stretch a membrane segment of area $A_{1}$ by the amount $\delta A_{1}$ is given by

$$
\delta F_{1}=A_{1} \frac{1}{2} K_{\mathrm{A}}\left(\delta A_{1} / A_{1}\right)^{2}
$$

where $K_{\mathrm{A}}$ is the area compressibility modulus which is an energy per unit area. If one now uses the relation (A1), one obtains the minimal segment size

$$
L_{1}=\left(K_{\mathrm{A}} / 4 \kappa \delta \bar{E}^{\circ}\right)^{1 / 2} N^{2} .
$$

Using the general relation $K_{\mathrm{A}}=c \kappa / a_{\text {mem }}^{2}$ for the area compressibility modulus where $c$ is a dimensionless coefficient, one obtains

$$
L_{1}=\left(c / 4 \delta \bar{E}^{\circ}\right)^{1 / 2} N^{2} / a_{\mathrm{mem}} .
$$

How long does it take to stretch a membrane segment of size $L_{1}$ ? The sound waves within the membrane are propagating modes with velocity $\sim \sqrt{K_{\mathrm{A}} / \rho_{\mathrm{A}}}$, where $\rho_{\mathrm{A}}$ is the mass density per unit area. Thus, it takes a time of the order of

$$
t_{\mathrm{s}} \sim\left(\rho_{\mathrm{A}} / K_{\mathrm{A}}\right)^{1 / 2} L_{1}=\left(\rho_{\mathrm{A}} / 4 \kappa \delta \bar{E}^{\circ}\right)^{1 / 2} N^{2}
$$

with $N=N_{\mathrm{b}}^{\mathrm{O}}$ as given by (2.14) in order to pull in the area of the neck by stretching the surrounding membrane. 
A2. FLATTENING OF SURROUNDING MATRIX.- The undulation modes of the membrane segment can be described in terms of the variable $\ell$ which measures the local displacement of the membrane from a flat reference state. A membrane segment of area $A_{1}=\pi L_{1}^{2}$ contains $\simeq A_{1} / a_{\text {mem }}^{2}$ undulation modes, $\tilde{\ell}$, which are the Fourier components of the displacement field $\ell$. The probability to find a certain configuration of the displacement field is given by the Boltzmann factor, $\exp [-\mathcal{H}\{\ell\} / T]$, with the effective Hamiltonian

$$
\mathcal{H}\{\ell\}=\int \frac{\mathrm{d}^{2} q}{(2 \pi)^{2}} \frac{1}{2}\left(\Sigma q^{2}+\kappa q^{4}\right)|\tilde{\ell}(\mathbf{q})|^{2} .
$$

The two parameters $\Sigma$ and $\kappa$ are the lateral tension and the bending rigidity, respectively.

Starting from this model, one can calculate the excess area $\Delta A_{1}$ stored in the undulations. As a result, one obtains [34]

$$
\frac{\Delta A_{1}(\Sigma)}{A_{1}}=\frac{T}{8 \pi \kappa} \ln \left(\frac{\Sigma / \kappa+q_{\max }^{2}}{\Sigma / \kappa+q_{\min }^{2}}\right)
$$

where $q_{\min }$ and $q_{\max }$ are appropriate spherical cutoffs for the absolute value, $q$, of the wavevector: $q_{\min } \leq q \leq q_{\max }$. The area pulled out by the lateral tension $\Sigma$ is $\delta A_{1}=\Delta A_{1}(0)-\Delta A_{1}(\Sigma)$ It then follows from (A7) that

$$
\begin{aligned}
\frac{\delta A_{1}(\Sigma)}{A_{1}} & =\frac{T}{8 \pi \kappa} \ln \left\{\left(\frac{q_{\max }}{q_{\min }}\right)^{2} \frac{\Sigma / \kappa+q_{\min }^{2}}{\Sigma / \kappa+q_{\max }^{2}}\right\} \\
& \approx \frac{T}{8 \pi \kappa} \ln \left\{1+\Sigma / \kappa q_{\min }^{2}\right\}
\end{aligned}
$$

where the asymptotic equality holds for $\Sigma / \kappa \ll q_{\max }^{2}$

As area is pulled out by the lateral tension, the free energy of the membrane segment changes according to

$$
\begin{aligned}
\delta F_{1} & =F_{1}(\Sigma)-F_{1}(0) \\
& \approx A_{1}(T / 2) \int \frac{\mathrm{d}^{2} q}{(2 \pi)^{2}} \ln \left(1+\Sigma / \kappa q^{2}\right)
\end{aligned}
$$

in the limit of large segment area $A_{1}$. An asymptotic expansion of the integral in (A9) leads to

$$
\delta F_{1}=A_{1}(T \Sigma / 8 \pi \kappa)\left\{-\ln \left(\Sigma / \kappa q_{\max }^{2}\right)+1+O\left(\Sigma / \kappa q_{\max }^{2}\right)\right\} .
$$

In the latter expression, the lateral tension $\Sigma$ may be expressed in terms of the area difference $\delta A_{1}$ by inverting the relation (A8). It is convenient to choose the cutoffs $q_{\max }=2 \sqrt{\pi} / a_{\mathrm{mem}}$ and $q_{\mathrm{min}}=2 \sqrt{\pi} / L_{1}$ with $A_{1}=\pi L_{1}^{2}$ as before. As a result, one obtains the excess free energy

$$
\delta F_{1} \approx \frac{1}{2} T \pi y\left\{-\ln \left(y / y_{\mathrm{sc}}\right)+1\right\}
$$

with

$$
y \equiv \exp \left(\frac{8 \pi \kappa}{T} \frac{\delta A_{1}}{A_{1}}\right)-1
$$

and $y_{\text {sc }} \equiv L_{1} / a_{\text {mem }}$ which applies as long as $y \ll y_{\text {sc }}$

In order to determine the minimal value for $L_{1}$, one has to insert the relations $\delta F_{1}=\Delta E^{\circ}$ and $\delta A_{1}=\pi N^{2}$ into (A11) and (A12). In the limit of small $y / y_{\mathrm{sc}}$, one then obtains the asymptotic behavior

$$
L_{1} \approx\left[8 \pi \kappa / T \ell \mathbf{n}\left(1+2 \delta E^{\circ} / \pi T\right)\right]^{1 / 2} N .
$$


Note that this value for $L_{1}$ has only a weak logarithmic dependence on $\delta E^{\circ}=2 \pi \kappa \delta \bar{E}^{\circ}$. Apart from this logarithmic factor, the behavior as given by (A13) can also be obtained directly from (A7) or (A8) with $\Delta A_{1} \simeq \pi N^{2}$ or $\delta A_{1}=\pi N^{2}$ provided one ignored the logarithmic factors in the latter equations.

How long does it take to flatten a membrane segment of size $L_{1}$ ? The undulations of a fluid membrane are overdamped modes which have a relaxation time $\sim \eta / \kappa q^{3}$ where $\eta$ is the (dynamic) viscosity of the aqueous solution surrounding the membrane [35]. The parameter $\eta$ has the units of time $\mathrm{x}$ energy/volume. Therefore, the perturbation induced by the transforming bud will spread over the membrane segment of size $L_{1}$ in the time

$$
t_{\mathrm{f}} \sim \eta L_{1}^{3} / \kappa \sim(8 \pi)^{3 / 2} \eta \kappa^{1 / 2} N^{3} / T^{3 / 2}
$$

where $N$ denotes the neck radius $N=N_{\mathrm{ib}}^{\mathrm{o}}$ of the incomplete bud and the logarithmic factor in (A13) has been omitted.

\section{References}

[1] For a review, see Lipowsky R., Nature 349 (1991) 475.

[2] Berndl K., Käs J., Lipowsky R., Sackmann E. and Seifert U., Europhys. Lett. 13 (1990) 659.

[3] Käs J. and Sackmann E., Biophys. J. 60 (1991) 1.

[4] Seifert U., Berndl K. and Lipowsky R., Phys. Rev. A 44 (1991) 1182.

[5] Miao L., Fourcade B., Rao M., Wortis M. and Zia R.K.P. Phys. Rev. A 43 (1991) 6843.

[6] Wiese W., Harbich W. and Helfrich W., J. Phys. France (submitted).

[7] The strong curvature in the neck region of the bud could induce the accummulation of impurities within this region as suggested by Fischer T.M., J. Phys. II France 2 (1992) 327. In such a situation, the shape transformation would induce the segregation of impurity molecules. This has to be distinguished from the novel mechanism discussed in the present work where the phase separation drives the shape transformation.

[8] Alberts B., Bray D., Lewis J., Raff M., Roberts K. and Watson J.D., Molecular Biology of the Cell (Garland Publishing, New York, 1988).

[9] Darnell J., Lodish H. and Baltimore D., Molecular Cell Biology (Freeman, New York, 1990).

[10] Pearse B.M.F. and Crowther R.A., Ann. Rev. Biophys. Biophys. Chem. 16 (1987) 49-68.

[11] Brodsky F.M., Science 242 (1988) 1396-1402.

[12] Oster G.F., Cheng L.Y., Moore H.-P.H. and Perelson A.S., J. theor. Biol. 141 (1989) 463.

[13] The phase separation of a two-component mixture could also lead to a striped phase within the membrane. The effect of such a periodic modulation on the membrane shape has been considered by Andelman D., Kawakatsu T. and Kawasaki K., Europhys. Lett. 19 (1992) 57.

[14] Helfrich W., Phys. Lett. 50 A (1974) 115.

[15] Strictly speaking, the incomplete bud becomes already unstable as soon as the energy barrier has been sufficiently reduced and $\Delta E / T=2 \pi \kappa \Delta \bar{E} / T$ becomes of order one. However, the difference between the corresponding value of $L$ and the limiting size $L^{\circ}$ is small for all parameter values considered in table I.

[16] San Miguel M., Grant M. and Gunton J.D., Phys. Rev. A 31 (1985) 1001.

[17] Huse D.A., Phys. Rev. B 34 (1986) 7845.

[18] Gebhardt C., Gruler H. and Sackmann E., Z. Naturforsch. 32c (1977) 581.

[19] Gaub H., Büschel R., Ringsdorf H. and Sackmann E., Chem. Phys. Lipids 37 (1985) 19.

[20] Sackmann E., Can. J. Phys. 68 (1990) 999.

[21] Vaz W.L., Melo E.C.C. and Thompson T.E., Biophys. J. 56 (1989) 869.

[22] Vist M.R. and Davis J.H., Biochem. 29 (1990) 451. 
[23] Needham D., McIntosh T.J. and Evans E., Biochem. 27 (1988) 4668.

[24] Bloom H., Evans E. and Mouritsen O.G., Quart. Rev. Biophys. 24 (1991) 293.

[25] Wu S.H. and McConnell H.M., Biochemistry 14 (1975) 847.

[26] Bloom M., Phys. Can. 48 (1992) 7-16.

[27] Duwe H.P., Kaes J. and Sackmann E., J. Phys. France 51 (1990) 945-962.

[28] Mutz M. and Helfrich W., J. Phys. France 51 (1990) 991-1002.

[29] For a review, see Clegg R.M. and Vaz W.L.C., Progress in Protein-Lipid Interactions, Watts and De Pont Eds. (Elsevier Science Publishers, 1985) pp. 173-229.

[30] Evans E. and Needham D., J. Phys. Chem. 91 (1987) 4219.

[31] Kaler E., Murthy A.K., Rodriguez B.E. and Zasadzinski J.A.N., Science 245 (1989) 1371.

[32] Heuser J. and Kirchhausen T., J. Ultrastr. Res. 92 (1985) 1-27.

[33] Stryer L., Biochemistry (Freeman, New York, 1988).

[34] Helfrich W. and Servuss R.M., Nuovo Cimento D 3 (1984) 137.

[35] Brochard F. and Lennon J.F., J. Phys. France 36 (1975) 1035. 